\title{
2-DISTANCE COLORINGS OF INTEGER DISTANCE GRAPHS
}

\author{
Brahim Benmedjdoub, Isma Bouchemakh \\ Faculty of Mathematics, Laboratory L'IFORCE \\ University of Sciences and Technology Houari Boumediene (USTHB) \\ B.P. 32 El-Alia, Bab-Ezzouar, 16111 Algiers, Algeria \\ e-mail: brahimro@hotmail.com \\ isma_bouchemakh2001@yahoo.fr \\ AND \\ ÉRIC SOPENA \\ Univ. Bordeaux, Bordeaux INP, CNRS, LaBRI, UMR5800 \\ F-33400 Talence, France \\ e-mail: Eric.Sopena@labri.fr
}

\begin{abstract}
A 2-distance $k$-coloring of a graph $G$ is a mapping from $V(G)$ to the set of colors $\{1, \ldots, k\}$ such that every two vertices at distance at most 2 receive distinct colors. The 2-distance chromatic number $\chi_{2}(G)$ of $G$ is then the smallest $k$ for which $G$ admits a 2 -distance $k$-coloring. For any finite set of positive integers $D=\left\{d_{1}, \ldots, d_{\ell}\right\}$, the integer distance graph $G=G(D)$ is the infinite graph defined by $V(G)=\mathbb{Z}$ and $u v \in E(G)$ if and only if $|v-u| \in D$. We study the 2-distance chromatic number of integer distance graphs for several types of sets $D$. In each case, we provide exact values or upper bounds on this parameter and characterize those graphs $G(D)$ with $\chi_{2}(G(D))=\Delta(G(D))+1$.
\end{abstract}

Keywords: 2-distance coloring, integer distance graph.

2010 Mathematics Subject Classification: 05C15, 05C12.

\section{REFERENCES}

[1] G. Agnarsson and M.M. Halldórsson, Coloring powers of planar graphs, SIAM J. Discrete Math. 16 (2003) 651-662. doi:10.1137/S0895480100367950 
[2] M. Bonamy, B. Lévêque and A. Pinlou, 2-distance coloring of sparse graphs, J. Graph Theory 77 (2014) 190-218.

doi:10.1002/jgt.21782

[3] M. Bonamy, B. Lévêque and A. Pinlou, Graphs with maximum degree $\Delta \geq 17$ and maximum average degree less than 3 are list 2-distance $(\Delta+2)$-colorable, Discrete Math. 317 (2014) 19-32.

doi:10.1016/j.disc.2013.10.022

[4] O.V. Borodin and A.O. Ivanova, 2-distance $(\Delta+2)$-coloring of planar graphs with girth six and $\Delta \geq 18$, Discrete Math. 309 (2009) 6496-6502. doi:10.1016/j.disc.2009.06.029

[5] T. Calamoneri, The $L(h, k)$-labelling problem: An updated survey and annotated bibliography, Comput. J. 54 (2011) 1344-1371. doi:10.1093/comjnl/bxr037

[6] Z. Dvořák, D. Král, P. Nejedlý and R. Škrekovski, Coloring squares of planar graphs with girth six, European J. Combin. 29 (2008) 838-849. doi:10.1016/j.ejc.2007.11.005

[7] A. Kemnitz and H. Kolberg, Coloring of integer distance graphs, Discrete Math. 191 (1998) 113-123. doi:10.1016/S0012-365X(98)00099-5

[8] F. Kramer and H. Kramer, Un probleme de coloration des sommets d'un graphe, C. R. Acad. Sci. Paris A 268 (1969) 46-48.

[9] F. Kramer and H. Kramer, A survey on the distance-colouring of graphs, Discrete Math. 308 (2008) 422-426. doi:10.1016/j.disc.2006.11.059

[10] K.-W. Lih and W.-F. Wang, Coloring the square of an outerplanar graph, Taiwanese J. Math. 10 (2006) 1015-1023. doi: $10.11650 /$ twjm/1500403890

[11] D.D.-F. Liu, From rainbow to the lonely runner: A survey on coloring parameters of distance graphs, Taiwanese J. Math. 12 (2008) 851-871. doi:10.11650/twjm/1500404981

[12] M. Voigt, Über die chromatische Zahl einer speziellen Klasse unendlicher Graphen, Ph.D. Thesis (Techn. Univ. Ilmenau, 1992).

[13] H. Walther, Über eine spezielle Klasse unendlicher Graphen, in: Graphentheorie, K. Wagner and R. Bodendiek (Ed(s)), (Bibl. Inst., Mannheim, 1990) 2, 268-295.

[14] W.-F. Wang and K.-W. Lih, Labeling planar graphs with conditions on girth and distance two, SIAM J. Discrete Math. 17 (2003) 264-275. doi: $10.1137 /$ S0895480101390448

[15] G. Wegner, Graphs with given diameter and a coloring problem (Technical Report, University of Dortmund, 1977).

doi:10.17877/DE290R-16496 
[16] X. Zhu, Circular chromatic number of distance graphs with distance sets of cardinality 3, J. Graph Theory 41 (2002) 195-207.

doi:10.1002/jgt.10062

Received 5 December 2016

Revised 12 November 2017

Accepted 13 November 2017 